\title{
Dust dynamic pressure and magnetopause displacement: reasons for non-detection
}

\author{
I. Mann ${ }^{1}$ and M. Hamrin ${ }^{2}$ \\ ${ }^{1}$ EISCAT Scientific Association, Kiruna, Sweden \\ ${ }^{2}$ Department of Physics, Umeå University, Umeå, Sweden \\ Correspondence to: I. Mann (ingrid.mann@eiscat.se)
}

Received: 31 May 2012 - Revised: 2 November 2012 - Accepted: 26 November 2012 - Published: 7 January 2013

\begin{abstract}
In a recent paper, Treumann and Baumjohann (2011) propose that the contribution of dust particles to the solar wind dynamic pressure can cause large compressions of the Earth's magnetopause and suggest that this occurs when Earth encounters meteoroid streams. In this paper we estimate the contribution from charged dust particles to the solar wind dynamical pressure, and we exclude that the dust associated to meteoroid streams can influence the extension of the magnetopause according to the proposed model. A sufficient coupling to the solar wind is only expected for so-called nanodust. However, the dynamic pressure of the nanodust is orders of magnitudes below that of the solar wind, making it unlikely that its variation can be observed in displacements of the magnetopause. We also discuss the equation that the authors use for estimating the extension of the Earth's magnetopause, and conclude that this is not applicable due to the large gyroradius of the nanodust. We finally note that an influence of dust on the extension of a magnetosphere might be quite possible in other astrophysical systems and based on other processes.
\end{abstract}

Keywords. Interplanetary physics (Interplanetary dust; Solar wind plasma) - Magnetospheric physics (Magnetopause, cusp, and boundary layers)

\section{Introduction}

The Earth's magnetopause plays an important role for the complex interactions between the Earth's magnetosphere and the Sun and the interplanetary medium. The resulting processes have important consequences for the geomagnetic environment of the Earth and ultimately influence the Earth's atmosphere. Magnetospheres are also observed around other planets of the solar system, and space measurements permit close studies of the processes that influence the magnetospheres. Mercury, for instance, being closest to the Sun, is especially prone to the changing solar wind conditions and magnetic field variations (Luhmann et al., 1998; Slavin et al., 2007). The physics of magnetospheres is also considered for studying the atmospheres of extra-solar planets (see e.g. Reiners and Christensen, 2010). Naturally, the Earth's magnetosphere is best studied both from space and from the ground. The overall changes of the magnetosphere are apparent in the variations of the Earths magnetopause, i.e. the boundary between the terrestrial magnetic field and the solar wind. While the overall position and shape of the Earth's magnetopause is primarily determined by a pressure balance between the solar wind dynamic pressure and Earth's magnetic pressure, there is a huge number of parameters and processes that can influence the magnetopauses in general.

The Earth's magnetopause has been well observed and described with different models. Many of them are parameterized by the solar wind dynamic pressure and the northsouth component of the interplanetary magnetic field (IMF) (Šafránková et al., 2002). Over the years, various processes modifying the magnetopause position and shape have been discussed. Already in 1970, Aubry et al. (1970) found that the orientation of the IMF influences the magnetopause position. For southward IMF, dayside reconnection is expected. This results in an erosion of the magnetopause due to magnetic flux being transferred from the dayside to the nightside (Shue et al., 2000). Lobe reconnection, on the other hand, would imply a reversed process, with magnetic flux being transferred from the nightside to the dayside (Shue and Song, 2002). Šafránková et al. (2002) used observed magnetopause crossings from several space missions for a 
comparison between different models. More recently, the five spacecraft of the THEMIS mission have observed around 6500 magnetopause crossings, and these data were used to investigate the dependence of the magnetopause location on the angle between the interplanetary magnetic field and solar wind velocity vectors (i.e. IMF cone angle) (Dusik et al., 2010). Dusik et al. (2010) note that the dependence of the magnetopause location on the solar wind dynamic pressure is stronger than predicted by the models.

In a recent work, Treumann and Baumjohann (2011) consider the contribution of the dust component to the solar wind dynamic pressure and suggest that enhanced dust number density, observed when Earth crosses meteoroid streams, raises the contribution of the dust component to the solar wind dynamical pressure to an extent that this is observed in variations of the extension of the magnetopause. While the presence of dust particles is not frequently discussed in the context of the planetary magnetospheres, the influence of interstellar dust dynamic pressure is considered by Holzer (1989), for instance, when describing the extension of the heliosphere. Its outer boundary, the heliopause, builds up as a result of the pressure balance between the solar wind and the interstellar gas. Holzer (1989) finds, however, that the dust component is of minor importance.

In this paper we consider the suggestion by Treumann and Baumjohann (2011) based on our current knowledge of the dust in the solar system near the Earth's orbit. We start by introducing the model for describing the extension of the magnetopause and revisit the formula and figure given by the authors, since we found some difficulties in understanding their presentation. We then discuss the dust components in the interplanetary medium and their possible contribution to the solar wind dynamic pressure. We also discuss the dust trajectories in order to check whether they can be described in the fluid approach that the Treumann and Baumjohann (2011) model is based upon. The paper ends with a summary.

\section{An extended version of the Treumann and Baumjo- hann model}

In a simple model, the pressure balance that determines the magnetopause can be expressed as (Spreiter et al., 1966)

$k \rho V^{2} \cos ^{2} \theta=\frac{(2 f \boldsymbol{B})^{2}}{2 \mu_{0}}$,

where $\rho=N M$ and $V$ are the solar wind mass density and speed, $\boldsymbol{B}$ is the magnetospheric magnetic field at the location of the magnetopause, $\theta$ is the angle between the magnetopause normal and the Sun-Earth line, $f$ is a parameter describing the compression of the terrestrial magnetic field, and $k$ is a parameter describing the reduction of the dynamic pressure by flow diversion around the magnetopause.

The case $\theta=0$ describes the sub-solar magnetopause stagnation point, $R$. Assuming a dipolar magnetic field, i.e. $B \sim$ $r^{-3}$, we obtain

$R=C B_{0}\left[\sum_{\mathrm{i}} N_{\mathrm{i}} m_{\mathrm{i}} V^{2}\right]^{-\frac{1}{6}}$,

where the solar wind mass density should be summed over all relevant particle species, $C$ is a constant, and $B_{0}$ is the magnetic field at the surface of the Earth.

Treumann and Baumjohann (2011) discuss the balance of the dynamic pressure and the magnetic pressure in the presence of dust assuming three particle species: electrons, protons and dust. In the present article we also include alpha particles $(\mathrm{He}++)$ and obtain the solar wind dynamic pressure:

$\sum_{\mathrm{i}} N_{\mathrm{i}} m_{\mathrm{i}} V^{2}=M N V^{2}, \quad i=e, p, \alpha, d$

with the components electrons $(e)$, protons $(p)$, alpha particles $(\alpha)$, and charged dust $(d) . N_{i}$ is the number density of the species, $m_{\mathrm{i}}$ the mass, and $V$ the solar wind speed under the assumption that the dust particles are coupled to the solar wind, i.e. move with the same bulk speed as the electrons, protons, and alpha particles. Assuming charge neutrality one gets

$N_{\mathrm{e}}=N_{\mathrm{p}}+Q_{\alpha} N_{\alpha}+Q_{\mathrm{d}} N_{\mathrm{d}}$,

where $Q_{\alpha}$ and $Q_{\mathrm{d}}$ are the alpha particle and the dust charges, respectively. Using Eqs. (3) and (4) one obtains

$$
\begin{aligned}
M N V^{2}= & {\left[1+\frac{m_{\mathrm{e}}}{m_{\mathrm{p}}}+\alpha_{\alpha} A_{\alpha}\left(1+\frac{m_{\mathrm{e}}}{m_{\mathrm{p}}} \frac{Q_{\alpha}}{A_{\alpha}}\right)+\right.} \\
& \left.+\alpha_{\mathrm{d}} A_{\mathrm{d}}\left(1+\frac{m_{\mathrm{e}}}{m_{\mathrm{p}}} \frac{Q_{\mathrm{d}}}{A_{\mathrm{d}}}\right)\right] m_{\mathrm{p}} N_{\mathrm{p}} V^{2},
\end{aligned}
$$

where $A_{\alpha, \mathrm{d}}=m_{\alpha, \mathrm{d}} / m_{\mathrm{p}}$ and $\alpha_{\alpha, \mathrm{d}}=N_{\alpha, \mathrm{d}} / N_{\mathrm{p}}$ are the mass ratio and the number density ratio for alpha and dust particles. Substituting into Eq. (2), we obtain an expression for the subsolar magnetopause stagnation point

$$
\begin{aligned}
R= & R_{\mathrm{p}}\left[1+\frac{m_{\mathrm{e}}}{m_{\mathrm{p}}}+\alpha_{\alpha} A_{\alpha}\left(1+\frac{m_{\mathrm{e}}}{m_{\mathrm{p}}} \frac{Q_{\alpha}}{A_{\alpha}}\right)+\right. \\
& \left.+\alpha_{\mathrm{d}} A_{\mathrm{d}}\left(1+\frac{m_{\mathrm{e}}}{m_{\mathrm{p}}} \frac{Q_{\mathrm{d}}}{A_{\mathrm{d}}}\right)\right]^{-\frac{1}{6}} \\
= & R_{\mathrm{p}}\left[1+\frac{m_{\mathrm{e}}}{m_{\mathrm{p}}}+S_{\alpha}+S_{\mathrm{d}}\right]^{-\frac{1}{6}},
\end{aligned}
$$

where $R_{\mathrm{p}}$ is the stagnation point for a solar wind only containing protons, and the second, third $\left(S_{\alpha}=\right.$ $\left.\alpha_{\alpha} A_{\alpha}\left(1+m_{\mathrm{e}} Q_{\alpha} /\left(m_{\mathrm{p}} A_{\alpha}\right)\right)\right)$, and forth term $\left(S_{\mathrm{d}}=\alpha_{\mathrm{d}} A_{\mathrm{d}}(1+\right.$ $\left.\left.m_{\mathrm{e}} Q_{\mathrm{d}} /\left(m_{\mathrm{p}} A_{\mathrm{d}}\right)\right)\right)$ on the right hand side describe the contributions from electrons, alpha particles, and dust, respectively. This description follows that by Treumann and Baumjohann (2011) except for the second and third terms on the right hand side. The contribution of alpha particle $\left(S_{\alpha}\right)$ was not included by Treumann and Baumjohann (2011). Moreover, the second 


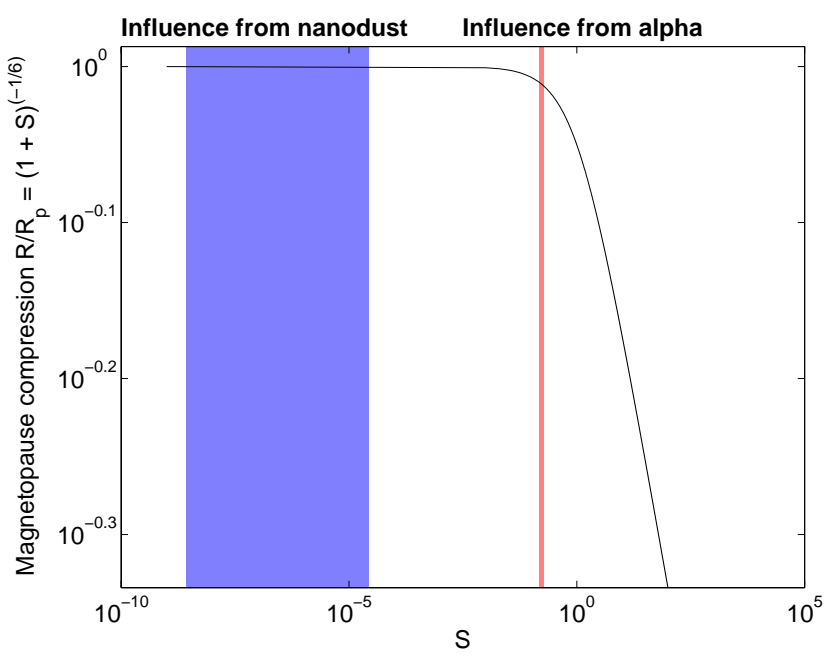

Fig. 1. The influence of alpha particles and dust on the position of the sub-solar magnetopause stagnation point. The red and blue regions show the relevant range for $S_{\alpha}$ and $S_{\mathrm{d}}$, respectively (see Eq. 6 and the discussion in the text). The compression of the magnetopause from the nanodust (blue region) is negligible for the location of the stagnation point, please also note limited applicability of Eq. (6). This figure is a modified version (as discussed in the text) of the Fig. 2 presented by Treumann and Baumjohann (2011).

term on the right hand side, $m_{\mathrm{e}} / m_{\mathrm{p}}$, is of the order $10^{-4}$, and was neglected.

By using the above equation (without the alpha particle contribution), Treumann and Baumjohann (2011) quantified the influence of the dust component in terms of a variation of the magnetopause sub-solar stagnation point distance with respect to $R_{\mathrm{p}}$. Figure 1 in this article is a modified version of Fig. 2 of Treumann and Baumjohann (2011). However, note the misprint in the caption of the original Fig. 2 of Treumann and Baumjohann (2011): the figure shows $R / R_{\mathrm{p}}=\left(1+S_{\mathrm{d}}\right)^{-1 / 6}$ versus $S_{\mathrm{d}}$. In order to be described within this equation, the components need to move with the solar wind flow. This is naturally the case for the solar wind helium ( $\alpha$ particles). Another component is the nanodust that is picked up by the solar wind. Its total momentum flux is, however, estimated to $\leq 10^{-6}$ of that of the solar wind near $1 \mathrm{AU}$ (Mann et al., 2011). We nonetheless insert the respective parameters here for illustration.

The evaluation of nanodust fluxes based on analysis of ongoing measurements with plasma wave instruments onboard the two Stereo spacecraft near 1 AU (Zaslavsky et al., 2012) provide a basis for our estimate. We assume the following typical parameters for nanodust at the orbit of Earth: $10^{-22} \mathrm{~kg}<m_{\mathrm{d}}<10^{-20} \mathrm{~kg}, 3 \times 10^{-7} \mathrm{~m}^{-3}<N_{\mathrm{d}}<3 \times$ $10^{-5} \mathrm{~m}^{-3}$, and $Q_{\mathrm{d}}=17 e$. Using these values, we find that the dust contribution to Eq. (6) is $3 \times 10^{-9}<S_{\mathrm{d}}<3 \times 10^{-7}$. Comparing to Fig. 2 of Treumann and Baumjohann (2011) (remember that this figure shows $R / R_{\mathrm{p}}=\left(1+S_{\mathrm{d}}\right)^{-1 / 6}$ versus $S_{\mathrm{d}}$ ), this corresponds to very small $S_{\mathrm{d}}$ values. By using

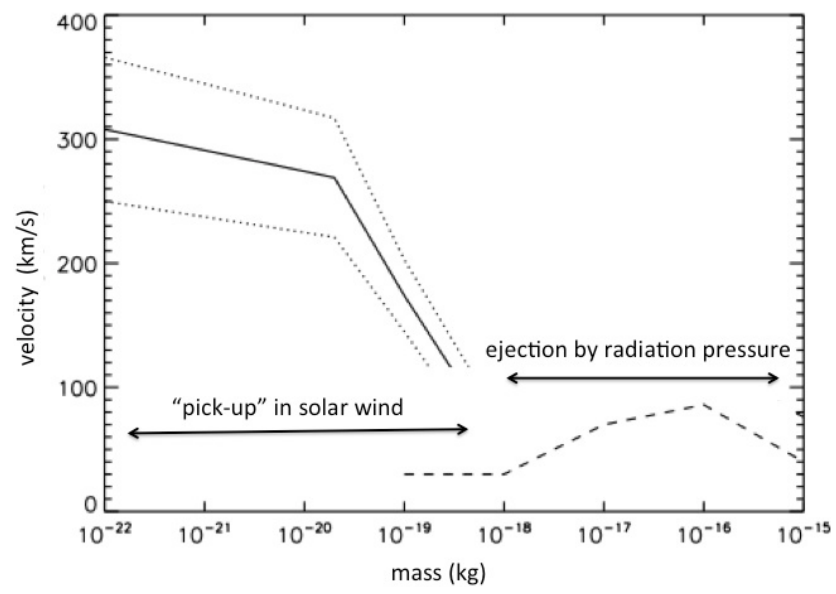

Fig. 2. The radial velocity component vs. mass for dust at $1 \mathrm{AU}$. The particle velocities for dust smaller than $10^{-19} \mathrm{~kg}$ are estimated based on a model of the formation and acceleration of nanodust in the inner solar system. To illustrate typical velocities of the larger dust with mass larger $10^{-19} \mathrm{~kg}$, we assume that it forms near $0.2 \mathrm{AU}$ and is ejected by radiation pressure, this gives an upper estimate. The dust larger than $10^{-15} \mathrm{~kg}$ is in Keplerian orbits. Further discussion can be found in the reference Mann et al. (2010b).

$0.038 N_{\mathrm{p}}<N_{\alpha}<0.048 N_{\mathrm{p}}$ (Klecker, 2009; Schwenn, 1983) for the alpha particle density, we get $0.15<S_{\alpha}<0.19$. In the simple model of Eq. (6), the dust contribution to the location of the sub-solar magnetopause stagnation point is hence many order of magnitudes smaller than the alpha particle contribution, and it is therefore negligible. This is illustrated in Fig. 1 where the region of influence from dust is highlighted in blue and from alpha particles in red.

\section{Comparison to dust parameters}

\subsection{Dust trajectories in the solar wind}

The basic assumption behind the proposed influence of dust on the magnetopause is that the dust carries a charge and that it is coupled to the solar wind. How does this compare to our knowledge of the dust in interplanetary space? Dust particles in the interplanetary medium acquire a surface potential relative to the surrounding on the order of several Volts, primarily as a result of photo ionization but also due to solar wind impact (see e.g. Kimura and Mann, 1998). Nonetheless, electromagnetic forces are typically small in comparison to gravity and radiation pressure, and the majority of the interplanetary dust particles move in some type of Keplerian orbits with electromagnetic force imposing a perturbation (Morfill and Grün, 1979). This picture changes when assuming nanodust particles with sizes $10 \mathrm{~nm}$ and smaller, as are identified in observations of the interstellar medium. If they also form in the interplanetary medium, they can be deflected and carried outward with the solar wind (Mann et al., 2007). Measurements 
onboard the Stereo spacecraft suggest the presence of such picked-up nanodust in the solar wind (Meyer-Vernet et al., 2010).

Figure 2 shows the velocities of dust near $1 \mathrm{AU}$ as a function of mass. One can estimate average values of the velocity of nanodust based on trajectory calculations when assuming a model of dust production inside 1 AU. Figure 2 shows such a model based on discussions in Mann et al. (2010b) in comparison to the velocities of the larger dust particles. One can see that the nanodust particles with masses $m \leq$ $10^{-19} \mathrm{~kg}$ have velocities $\sim 300 \mathrm{~km} \mathrm{~s}^{-1}$; the dust particles approximately in the mass interval $10^{-19} \mathrm{~kg} \leq m \leq 10^{-14} \mathrm{~kg}$ are significantly influenced by radiation pressure and depending on their optical properties can be repelled from the Sun. The shown velocity is obtained from a typical model of the optical properties of interplanetary dust. Larger dust particles (typically $m \geq 10^{-14} \mathrm{~kg}$ ) are mainly influenced by gravity, and their trajectories follow approximately Keplerian orbits. The dust flux near $1 \mathrm{AU}$ increases steeply with dust size, and the Stereo measurements show that the observed flux, $F$, is close to the curve $F \sim m^{-5 / 6}$ (Meyer-Vernet et al., 2010), but the larger dust particles do not influence the dynamic pressure since they have much smaller velocities.

\subsection{Dust pickup and fluid approach}

The deflection of the nanodust is similar, though not identical, to the behavior of pickup of ions in the solar wind, as described for instance by Luhmann, 2003). The pickup occurs for dust with surface charge to mass ratios $10^{-5} e / m_{\mathrm{p}}<$ $Q / m<10^{-4} e / m_{\mathrm{p}}$, which, assuming typical charging conditions in the interplanetary medium, are associated to dust particles of radii around $3 \mathrm{~nm}$ to $10 \mathrm{~nm}$ (Czechowski and Mann, 2010). The particles reach velocities of the order of the solar wind after traveling for a good fraction of one AU. Those particles that form closest to the Sun pass Earth's orbit with the highest speed. Trajectory calculations show that the nanodust moves in complex trajectories likened to a stretched spiral along a magnetic field line with gyration radii, $r_{\mathrm{g}}$, of the order of $1 / 100$ of an $\mathrm{AU}$, i.e. of the order of $10^{6} \mathrm{~km}$ in comparison of the extension of the magnetopause which is smaller than 15 Earth radii, i.e. smaller than $10^{5} \mathrm{~km}$.

While the exact value of the radius of gyration depends, for example, on the dust velocity perpendicular to the magnetic field (which is determined by the location where the nanodust forms) (Czechowski and Mann, 2012), one may evaluate the number based on the fact that $r_{\mathrm{g}} \sim m / Q$ to see that the ratio of $Q / m$ needs to be larger by about $10^{3}$ as compared to the values in the paragraph above for the fluid approach to be valid. These values of $Q / m$ do not occur for dust in the solar system (see e.g. Czechowski and Mann, 2010), but rather apply to heavy ions. In this context we point out that the influence of the gyration radius has been mentioned before in a different context. In a study of the solar wind interacting with Venus, Phillips et al. (1987) point out that a fluid treatment is not sufficient for describing the influence of pick-up ions to explain observational data, and that rather a hybrid or kinetic model is necessary.

\subsection{Earth-crossing meteoroid streams}

As a possible event during which the dust could cause the magnetopause compressions, Treumann and Baumjohann (2011) propose the encounters with meteoroid dust streams along Earth's orbit. One criticism to this model concerns the orbital properties of these meteoroid streams. The streams are observed as meteor showers, describing a number of meteoroids entering the Earth's atmosphere with approximately parallel trajectories (note that the term meteor denotes the brightness phenomenon, while meteoroid denotes the solid object moving in interplanetary space). The similar trajectories occur from meteoroids that move in interplanetary space with similar orbital parameters corresponding to an orbit that crosses Earth's orbit. Since the meteoroids follow bound orbits and hence the velocity of the meteoroids is below escape velocity $\left(42 \mathrm{~km} \mathrm{~s}^{-1}\right)$, they do not move with the solar wind speed, nor in the direction of the solar wind. Studies of the sporadic meteor orbital statistics could not provide any evidence for the detection of meteors that move faster than escape velocity (Hajdukova, 2008).

A further criticism bases on the total meteoroid mass that is associated with the meteor showers, since the vast majority of the mass that continuously enter Earth's atmosphere is associated to sporadic meteors (see e.g. Ceplecha et al., 1998) (note, it is not possible to derive absolute values of the fluxes in interplanetary space from the meteor observations). We also assume that production of nanodust or ions within the meteoroid streams is negligible. An estimate of the influence of cometary dust trails on the solar wind was carried out in a different context and showed that enhancements in density of free charges compared to the surrounding solar wind are marginal (Mann et al., 2010a).

\subsection{Summary and discussion}

We suggest that it is not reasonable to assume that Earthorbit-crossing meteoroid streams can influence the extension of the magnetopause as suggested by Treumann and Baumjohann (2011), nor does any other dust component in the solar wind. Since our reasoning against the proposed process is based on the characteristics of dust in the solar wind and since the effect is orders of magnitude below that of other processes, we presume that our result may hold in general for the magnetospheres of the other planets in the solar system. We suggest that variations in the solar wind composition play a more important role for the extension of the magnetopause than the dust does.

We note, however, that a dust component may also generate a dynamic pressure without being coupled to the solar wind. In the case of the solar system, the nanodust is picked 
up in the solar wind. Dust particles can also be accelerated by radiation pressure forces. Such dust is not coupled to the solar wind and has a different velocity. In the solar system, this is the case for larger dust grains (see Fig. 1). The radiation pressure force depends on dust properties, but also on the spectrum and brightness of the host star. The dust particles in disks around stars with higher photospheric temperature and higher UV flux reach considerably higher speed (Czechowski and Mann, 2007). Such a dust component may influence the position and shape of magnetospheres in other planetary systems. Systems with strong stellar UV brightness and/or strong stellar wind may be an interesting topic for future research.

While the dynamics of nanodust in the solar wind is similar to the that of pick-up ions, the radii of gyration are larger than those of the pick-up ions, and this prevents us from using the fluid approach when estimating the extension of the Earth's magnetopause. We hence conclude that the formula suggested by Treumann and Baumjohann (2011) is not adequate for describing the influence of a dust component on scales of the order of the extension of the Earth's magnetosphere and smaller.

Acknowledgements. This paper was prepared while IM stayed as guest professor at Umeå University and this support is greatly acknowledged. The authors also acknowledge helpful discussion with Rudolf Treumann on his related work.

Topical Editor R. Nakamura thanks Y.-D. Jia and one anonymous referee for their help in evaluating this paper.

\section{References}

Aubry, M., Russell, C., and Kivelson, M.: Inward motion of magnetopause before a substorm, J. Geophys. Res., 75, 7018, doi:10.1029/JA075i034p07018, 1970.

Ceplecha, Z., Borovicka, J., Elford, W., Revelle, D., Hawkes, R., Porubcan, V., and Simek, M.: Meteor phenomena and bodies, Space Sci. Rev., 84, 327-471, doi:10.1023/A:1005069928850, 1998.

Czechowski, A. and Mann, I.: Collisional vaporization of dust and production of gas in the beta Pictoris dust disk, Astrophys. Journal, 660, 1541-1555, doi:10.1086/512965, 2007.

Czechowski, A. and Mann, I.: Formation and acceleration of nano dust in the inner heliosphere, Astrophys. Journal, 714, 89-99, doi:10.1088/0004-637X/714/1/89, 2010.

Czechowski, A. and Mann, I.: Nanodust Dynamics in Interplanetary Space, in: Astrophysics and Space Science Library, edited by: Mann, I., Meyer-Vernet, N., and Czechowski, A., vol. 385 of Astrophysics and Space Science Library, p. 47, doi:10.1007/9783-642-27543-2_4, 2012.

Dusik, S., Granko, G., Safrankova, J., Nemecek, Z., and Jelinek, K.: IMF cone angle control of the magnetopause location: Statistical study, Geophys. Res. Lett., 37, L19103, doi:10.1029/2010GL044965, 2010.

Hajdukova Jr., M.: Meteors in the IAU meteor data center on hyperbolic orbits, Earth moon and planets, 102, 67-71, doi:10.1007/s11038-007-9171-5, Meteoroids Conference 2007, CosmoCaixa Sci Museum, Barcelona, Spain, 11-15 June 2007, 2008.

Holzer, T.: Interaction between the solar-wind and the interstellarmedium, Annu. Rev. Astron. Astrophys., 27, 199-234, doi:10.1146/annurev.astro.27.1.199, 1989.

Kimura, H. and Mann, I.: The electric charging of interstellar dust in the solar system and consequences for its dynamics, Astrophys. Journal, 499, 454-462, doi:10.1086/305613, 1998.

Klecker, B.: Interplanetary Particles and Magnetic Fields, in: Landolt-Börnstein - Group VI Astronomy and Astrophysics Numerical Data and Functional Relationships in Science and Technology Volume, edited by: Trümper, J. E., p. 436, doi:10.1007/978-3-540-88055-4_33, 2009.

Luhmann, J.: Characteristics of scatter-free behavior of heliospheric pickup ions, Astrophys. Journal, 592, 1241-1251, doi:10.1086/375770, 2003.

Luhmann, J., Russell, C., and Tsyganenko, N.: Disturbances in Mercury's magnetosphere: Are the mariner 10 "substorms" simply driven?, J. Geophys. Res., 103, 9113-9119, doi:10.1029/97JA03667, 1998.

Mann, I., Murad, E., and Czechowski, A.: Nanoparticles in the inner solar system, Planet. Space Sci., 55, 1000-1009, doi:10.1016/j.pss.2006.11.015, 2nd Annual Meeting of the Asia-Oceania-Geosciences-Society, Singapore, Singapore, 2024 June 2005, 2007.

Mann, I., Czechowski, A., and Meyer-Vernet, N.: Dust In The Interplanetary Medium - Interactions With The Solar Wind, in: Twelfth international solar wind conference, edited by: Maksimovic, M., Issautier, K., MeyerVernet, N., Moncuquet, M., and Pantellini, F, vol. 1216 of AIP Conference Proceedings, pp. 491496, Amer inst physics, doi:10.1063/1.3395911, 12th International Solar Wind Conference, St Milo, France, 21-26 June 2009, 2010a.

Mann, I., Czechowski, A., Meyer-Vernet, N., Zaslavsky, A., and Lamy, H.: Dust in the interplanetary medium, Plasma Physics and Controlled Fusion, 52, doi:10.1088/07413335/52/12/124012, 37th European-Physical-Society Conference on Plasma Physics, Dublin City Univ, Helix Arts Ctr, Dublin, Ireland, 22-25 June 2010, 2010 b.

Mann, I., Pellinen-Wannberg, A., Murad, E., Popova, O., MeyerVernet, N., Rosenberg, M., Mukai, T., Czechowski, A., Mukai, S., Safrankova, J., and Nemecek, Z.: Dusty Plasma Effects in Near Earth Space and Interplanetary Medium, Space Sci. Rev., 161, 1-47, doi:10.1007/s11214-011-9762-3, 2011.

Meyer-Vernet, N., Czechowski, A., Mann, I., Maksimovic, M., Lecacheux, A., Goetz, K., Kaiser, M. L., Cyr, O. C. S., Bale, S. D., and Le Chat, G.: Detection of fast nanoparticles in the solar wind, in: Twelfth international solar wind conference, edited by: Maksimovic, M., Issautier, K., MeyerVernet, N., Moncuquet, M., and Pantellini, F, vol. 1216 of AIP Conference Proceedings, pp. 502-505, Amer inst physics, doi:10.1063/1.3395912, 12th International Solar Wind Conference, St Milo, France, 21-26 June 2009, 2010.

Morfill, G. E. and Grün, E.: The motion of charged dust particles in interplanetary space-II. Interstellar grains, Planet. Space Sci., 27, 1283-1292, doi:10.1016/0032-0633(79)90106-5, 1979.

Phillips, J., Luhmann, J., Russell, C., and Moore, K.: Finite larmor radius effect on ion pickup at Venus, J. Geophys. Res., 92, 9920- 
9930, doi:10.1029/JA092iA09p09920, 1987.

Reiners, A. and Christensen, U. R.: A magnetic field evolution scenario for brown dwarfs and giant planets, Astron. Astrophys., 522, doi:10.1051/0004-6361/201014251, 2010.

Šafránková, J., Něemecěk, Z., Dušík, Š., Přrech, L., Sibeck, D. G., and Borodkova, N. N.: The magnetopause shape and location: a comparison of the Interball and Geotail observations with models, Ann. Geophys., 20, 301-309, doi:10.5194/angeo20-301-2002, 2002.

Schwenn, R.: The average solar wind in the inner heliosphere: Structures and slow variations, in: NASA Conference Publication, vol. 228 of NASA Conference Publication, pp. 489-507, 1983.

Shue, J. and Song, P.: The location and shape of the magnetopause, Planet. Space Sci., 50, 549-558, doi:10.1016/S00320633(02)00034-X, 2002.

Shue, J., Russell, C., and Song, P.: Shape of the low-latitude magnetopause: Comparison of models, in: Coordinated Measurements of Magnetospheric Processes, edited by: Russell, C. T., vol. 25 of Advances in space research-series, pp. 1471-1484, Int. Union Geodesy \& Geophys, Int. Assoc. Geomagnetism \& Aeron.; Comm. Space Res., Pergamon press ltd, doi:10.1016/S02731177(99)00658-4, 2000.
Slavin, J. A., Krimigis, S. M., Acuna, M. H., Anderson, B. J., Baker, D. N., Koehn, P. L., Korth, H., Livi, S., Mauk, B. H., Solomon, S. C., and Zurbuchen, T. H.: MESSENGER: Exploring mercury's magnetosphere, Space Sci. Rev., 131, 133-160, doi:10.1007/s11214-007-9154-x, 2007.

Spreiter, J., Summers, A., and Alksne, A.: Hydromagnetic flow around magnetosphere, Planet. Space Sci., 14, 223, doi:10.1016/0032-0633(66)90124-3, 1966.

Treumann, R. A. and Baumjohann, W.: Magnetopause displacements: the possible role of dust, Ann. Geophys., 29, 2219-2223, doi:10.5194/angeo-29-2219-2011, 2011.

Zaslavsky, A., Meyer-Vernet, N., Mann, I., Czechowski, A., Issautier, K., Le Chat, G., Pantellini, F., Goetz, K., Maksimovic, M., Bale, S. D., and Kasper, J. C.: Interplanetary dust detection by radio antennas: mass calibration and fuxes measured by STEREO/WAVES, J. Geophys. Res., 117, A05102, in press, 2012. 